\title{
Spin dynamics of a magnetic Weyl semimetal $\mathrm{Sr}_{1-x} \mathrm{Mn}_{1-y} \mathrm{Sb}_{2}$
}

\author{
Zhengwei Cai, ${ }^{1}$, | Song Bao, ${ }^{1,}$ | Wei Wang, ${ }^{1}$, Zhen Ma, ${ }^{1}$ Zhao-Yang Dong, ${ }^{2}$ Yanyan Shangguan, ${ }^{1}$ \\ Jinghui Wang, ${ }^{1}$ Kejing Ran, ${ }^{1}$ Shichao Li, ${ }^{1}$ Kazuya Kamazawa, ${ }^{3}$ Mitsutaka Nakamura, ${ }^{4}$ \\ Devashibhai Adroja, ${ }^{5,6}$ Shun-Li Yu, ${ }^{1,7}$, † Jian-Xin Li, ${ }^{1,7}$, 用 and Jinsheng Wen ${ }^{1,7, \S}$ \\ ${ }^{1}$ National Laboratory of Solid State Microstructures and Department of Physics, Nanjing University, Nanjing 210093, China \\ ${ }^{2}$ Department of Applied Physics, Nanjing University of Science and Technology, Nanjing 210094, China \\ ${ }^{3}$ Neutron Science and Technology Center, Comprehensive Research \\ Organization for Science and Society (CROSS), Tokai, Ibaraki 319-1106, Japan \\ ${ }^{4}$ J-PARC Center, Japan Atomic Energy Agency (JAEA), Tokai, Ibaraki 319-1195, Japan \\ ${ }^{5}$ ISIS Facility, Rutherford Appleton Laboratory, Chilton, Didcot, Oxon OX11 0QX, United Kingdom \\ ${ }^{6}$ Highly Correlated Matter Research Group, Physics Department, \\ University of Johannesburg, P.O. Box 524, Auckland Park 2006, South Africa \\ ${ }^{7}$ Collaborative Innovation Center of Advanced Microstructures, Nanjing University, Nanjing 210093, China
}

\begin{abstract}
Dirac matters provide a platform for exploring the interplay of their carriers with other quantum phenomena. $\mathrm{Sr}_{1-x} \mathrm{Mn}_{1-y} \mathrm{Sb}_{2}$ has been proposed to be a magnetic Weyl semimetal and provides an excellent platform to study the coupling between Weyl fermions and magnons. Here, we report comprehensive inelastic neutron scattering (INS) measurements on single crystals of $\mathrm{Sr}_{1-x} \mathrm{Mn}_{1-y} \mathrm{Sb}_{2}$, which have been well characterized by magnetization and magnetotransport measurements, both of which demonstrate that the material is a topologically nontrivial semimetal. The INS spectra clearly show a spin gap of $\sim 6 \mathrm{meV}$. The dispersion in the magnetic $\mathrm{Mn}$ layer extends up to about $76 \mathrm{meV}$, while that between the layers has a narrow band width of $6 \mathrm{meV}$. We find that the linear spin-wave theory using a Heisenberg spin Hamiltonian can reproduce the experimental spectra with the following parameters: a nearest-neighbor $\left(S J_{1} \sim 28.0 \mathrm{meV}\right)$ and next-nearest-neighbor in-plane exchange interaction $\left(S J_{2} \sim 9.3 \mathrm{meV}\right)$, interlayer exchange coupling $\left(S J_{c} \sim-0.1 \mathrm{meV}\right)$, and spin anisotropy constant $(S D \sim-0.07 \mathrm{meV})$. Despite the coexistence of Weyl fermions and magnons, we find no clear evidence that the magnetic dynamics are influenced by the Weyl fermions in $\mathrm{Sr}_{1-x} \mathrm{Mn}_{1-y} \mathrm{Sb}_{2}$, possibly because that the Weyl fermions and magnons reside in the $\mathrm{Sb}$ and $\mathrm{Mn}$ layers separately, and the interlayer coupling is weak due to the quasi-two-dimensional nature of the material, as also evident from the small $S J_{c}$ of $-0.1 \mathrm{meV}$.
\end{abstract}

\section{INTRODUCTION}

Dirac semimetals are featured by two doublydegenerate linear bands crossing at the Dirac point $t^{1 / 3}$. The low-energy quasiparticle excitations near the Dirac point can be described by the $4 \times 4$ Dirac equation. In recent years, they have attracted a lot of attention because of their numerous exotic physical properties, such as high mobility $\frac{\sqrt[4]{6}}{6}$, nontrivial $\pi$ Berry phase ${ }^{7 \sqrt[9]{9}}$, and chiral-anomaly-induced negative magnetoresistance ${ }^{9}$ 11. Magnetic Dirac semimetals are particularly intriguing as they not only exhibit these fascinating phenomena but also offer a platform for investigating the interplay between Dirac fermions and spin dynamics. In this regard, the antiferromagnetic 112-type ternary compounds $\mathrm{AMnPn}_{2}$ (A represents alkali- or rare-earth elements such as $\mathrm{Sr}, \mathrm{Ca}, \mathrm{Ba}, \mathrm{Yb}$, or $\mathrm{Eu}$, and $\mathrm{Pn}=\mathrm{Sb}, \mathrm{Bi}$ ) have been studied extensively 12 . $\mathrm{SrMnBi}_{2}$ was initially reported to be such a material 13 . It was shown that the linearly dispersing $p$ bands of the Bi atoms formed highly anisotropic two-dimensional Dirac structure near the Fermi level, while the Mn bands were well below the Fermi level with binding energy larger than $2 \mathrm{eV}$ and thus were localized $13|14| 16] 19$. The localized divalent $\mathrm{Mn}\left(3 d^{5}\right.$, spin $S=5 / 2$ ) with a magnetic moment of magnitude $\sim 3.7 \mu_{\mathrm{B}}$ formed an antiferromagnetic structure below the Néel temperature $T_{\mathrm{N}}$ of $\sim 300 \mathrm{~K}^{20 \mid 21}$. Similar results with
Dirac fermions and magnons in the Bi- and Mn-square layers respectively were found by replacing $\mathrm{Sr}$ with $\mathrm{Ca}$ (Refs 16, 17, 20 23), Ba (Refs 24 and 25), Yb (Refs 2630), or Eu (Refs 31 33). However, in the $\mathrm{AMnBi}_{2}$ materials, the strong spin-orbital coupling (SOC) of $\mathrm{Bi}$ atoms might induce a gap that prevented the formation of the Dirac point, and made the fermions massive $e^{13|30| 34}$. One way to avoid the SOC-induced electronic gap opening and preserve the Dirac band structure is to replace $\mathrm{Bi}$ with lighter elements that have weaker SOC, e.g., Sb (Ref. 35). As a consequence, a number of $\mathrm{AMnSb}_{2}$-type materials where A represents Ca (Ref. [36), Sr (Refs 37-40), $\mathrm{Ba}$ (Refs 35, 41, and 42), or $\mathrm{Yb}$ (Refs 43 and 44) have been discovered.

Among these materials, $\mathrm{Sr}_{1-x} \mathrm{Mn}_{1-y} \mathrm{Sb}_{2}$ with $\mathrm{Sr}$ and $\mathrm{Mn}$ deficiencies stood out. It was suggested to be a magnetic Weyl semimetal which breaks the time-reversal symmetry $3745 / 46$, as opposed to non-magnetic ones that break the spatial inversion symmetry 4749 . Similar to $\mathrm{AMnBi}_{2}$-type magnetic Dirac materials, $\mathrm{Sr}_{1-x} \mathrm{Mn}_{1-y} \mathrm{Sb}_{2}$ was shown to exhibit fascinating properties that originated from the two-dimensional Sb and Mn square nets [Fig. 1(a)] (Ref. 37). Furthermore, the reduced size of the $\mathrm{SOC}$ because of the lighter mass of $\mathrm{Sb}$ as compared to $\mathrm{Bi}$ resulted in a nearly massless topological fermion behavior $\left(m^{*}=0.04-0.05 m_{0}\right.$, where $m^{*}$ and $m_{0}$ are the effective and free-electron masses, respectively) ${ }^{37}$. More importantly, it was shown that below the $T_{\mathrm{N}}$ of $304 \mathrm{~K}$, 
the material underwent a transition to a canted antiferromagnetic phase with a ferromagnetic component of $0.2 \mu_{\mathrm{B}}$ (Ref. 37). This small but finite ferromagnetic moment was proposed to break the time-reversal symmetry, lift the degeneracy of the electron bands, and lead to the long-sought magnetic Weyl semimetal state $26137 / 45$.

However, despite extensive studies on the aforementioned materials, whether and how the topological ferminons interplay with the magnons are still unsettled issues. In $\mathrm{CaMnBi}_{2}$, it was shown that there was a weak resistivity anomaly at the $T_{\mathrm{N}}$, indicative of a coupling between the Dirac bands and the magnetic ground state $e^{20}$, but no such anomaly was found in other reports2223. A two-magnon Raman scattering study of $\mathrm{CaMnBi}_{2}$ and $\mathrm{SrMnBi}_{2}$ indicated that Dirac carriers significantly enhanced the exchange coupling between the magnetic layers $\left(J_{c}\right)$, and a spin-fermion model showed that this in turn drove a charge-gap opening along the Dirac locus 50 . However, an inelastic neutron scattering (INS) study suggested that the $J_{c}$ estimated from the Raman measurements might have been exaggerated due to the neglecting of the single-ion spin anisotropy $\underline{\sqrt{50}}$, and instead it was found that the Dirac fermions did not affect the spin dynamics at all21. In $\mathrm{YbMnBi}_{2}$, nearly wave-vector independent damping in the INS spectra was taken as a signature of the spin-fermion coupling $\underline{51}$. In contrast, another INS work did not detect any anomalous features in the magnetic spectra, implying the absence of the coupling between magnons and topological fermions ${ }^{34}$.

In this work, we have carried out INS together with magnetization and magnetotransport measurements on single crystals of $\mathrm{Sr}_{1-x} \mathrm{Mn}_{1-y} \mathrm{Sb}_{2}$. We observe strong de Haas van Alphen (dHvA) and Shubnikov de Hass (SdH) quantum oscillations at $2 \mathrm{~K}$, from which we find topological fermions with $m^{*}=0.10 m_{0}$ and a nontrivial Berry phase, consistent with this material being a magnetic Weyl semimeta 37 . Our INS study reveals clear magnetic spectra dispersing from $6 \mathrm{meV}$ and extending up to $\sim 76 \mathrm{meV}$. The spin-wave spectra can be well described by a localized Heisenberg model with a nearest-neighbor$\left(S J_{1} \sim 28.0 \mathrm{meV}\right)$ and next-nearest-neighbor in-plane exchange interaction $\left(S J_{2} \sim 9.3 \mathrm{meV}\right)$, and interlayer exchange coupling $\left(S J_{c} \sim-0.1 \mathrm{meV}\right)$, along with a spin anisotropy term $(S D \sim-0.07 \mathrm{meV})$. From our results, we do not observe signatures that the magnetic dynamics are affected by the topological fermions.

\section{EXPERIMENTAL DETAILS}

Single crystals of $\mathrm{Sr}_{1-x} \mathrm{Mn}_{1-y} \mathrm{Sb}_{2}$ were grown using $\mathrm{Sb}$ as the self flux, with an initial atomic ratio of $\mathrm{Sr}: \mathrm{Mn}: \mathrm{Sb}=$ 1:1:4 for the raw materials. An alumina crucible containing the raw materials was sealed under Ar atmosphere in a quartz tube. The sealed ampule was heated up to $850{ }^{\circ} \mathrm{C}$, followed by a slow cooling at a rate of $1{ }^{\circ} \mathrm{C} / \mathrm{h}$ and centrifuged at $660{ }^{\circ} \mathrm{C}$. Single crystals with size up to $7 \times 5 \times 3 \mathrm{~mm}^{3}$ were so obtained, and some of the crystals are shown in Fig. 1(b). The composition of the single crystals was measured with energy dispersive spectroscopy (EDS) in an energy analyser (EX-250, Horiba) equipped in a scanning tunneling microscope (S-3400N II, Hitachi). Orientations of the crystals were determined by a single-crystal X-ray diffractometer (D8 Discover, Bruker) and a backscattering Laue X-ray diffractometer (LAUESYS, Photonic Science). Magnetization and resistivity measurements were conducted in a physical property measurement system (PPMS-9T, Quantum Design).

For INS experiments, single crystals were glued on aluminum plates by hydrogen-free Cytop grease. These crystals were well coaligned using a backscattering Laue X-ray diffractometer. Time-of-flight INS experiments were performed on 4 SEASONS located at J-PARC Center in Japan ${ }^{52}$, and MERLIN at ISIS Facility in the United Kingdom. We coaligned 27 pieces of single crystals weighed about $3.6 \mathrm{~g}$ in total for the experiment on 4 SEASONS, and 16 pieces weighed about $4.2 \mathrm{~g}$ in total for the experiment on MERLIN. The single crystals on the plates were mounted in the $(H, 0, L)$ plane, with the [010] direction aligned in the vertical direction. The assembly was loaded into a closed-cycle refrigerator. Both 4SEASONS and MERLIN were operated in a multiple- $E_{i}$ (incident energy) mode. For the experiment on 4SEASONS, we used a set of $E_{i}=100.1$ (primary), 47.8, and $18.3 \mathrm{meV}$ and a chopper frequency of $300 \mathrm{~Hz}$. We set the angle of the neutron beam direction parallel to the $c$ axis to be zero. Data were collected at $6 \mathrm{~K}$ by rotating the sample about the [010] direction with a range of $60^{\circ}$ in a $0.3^{\circ}$ step. On MERLIN, data were collected in the range of $90^{\circ}$ in a $1^{\circ}$ step at $6 \mathrm{~K}$, with a set of $E_{i}=90.2$ (primary), 31.8, and $16.0 \mathrm{meV}$ and a chopper frequency of $300 \mathrm{~Hz}$. We also conducted neutron scattering measurements at elevated temperatures on both spectrometers to investigate the temperature evolution of magnetic Bragg peaks. The wave vector $\boldsymbol{Q}$ was described by $(H K L)$ reciprocal lattice unit (rlu) of $\left(a^{*}, b^{*}, c^{*}\right)=(2 \pi / a, 2 \pi / b, 2 \pi / c)$. Although there is a small orthorhombicity suggested in Ref. 37, we used a tetragonal notation with $a \approx b=4.4342 \AA$, and $c=23.1359 \AA$. Note that we used the longest axis as $c$, which is the same as that in Refs 21 and 34 but different from that in Refs 37 and 40. In this case, our (001) corresponds to their (100).

\section{RESULTS}

\section{A. Sample Characterizations}

We checked the orientation of the $\mathrm{Sr}_{1-x} \mathrm{Mn}_{1-y} \mathrm{Sb}_{2}$ single crystals with single-crystal and backscattering Laue X-ray diffractometers, and the results are shown in Fig. 1(c) and (d), respectively. As shown in Fig.11(a), the crystal has a layered structure with $\mathrm{Sb}$ and Mn atoms forming square nets12137. The crystal naturally cleaves along the $c$ axis, so the cleavage plane can be nicely in- 


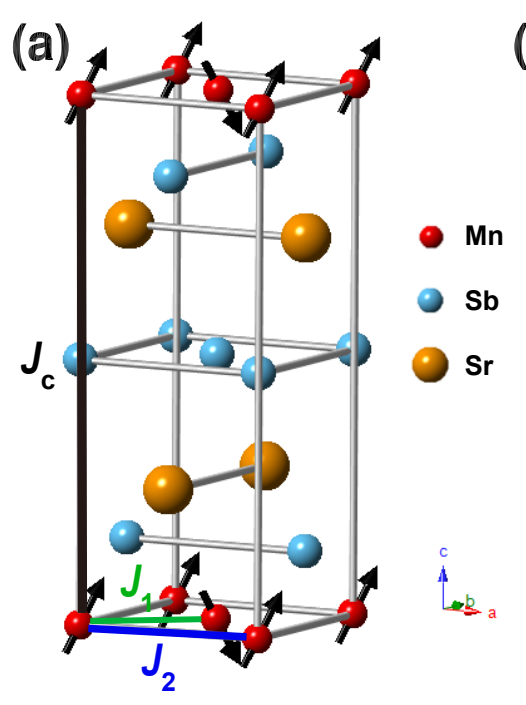

(b)

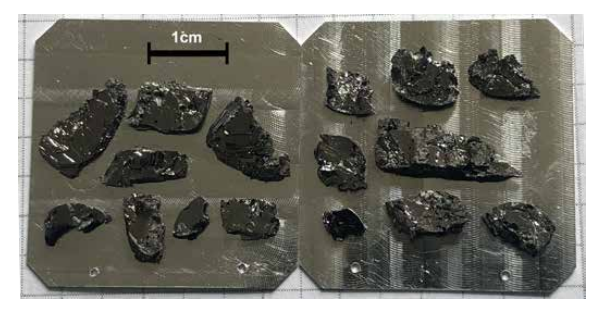

(c)

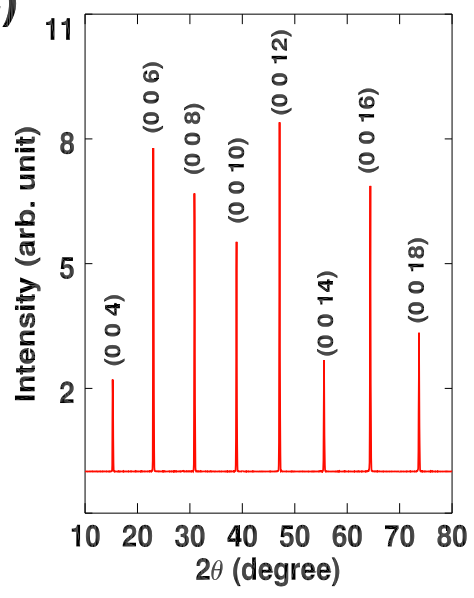

(d)

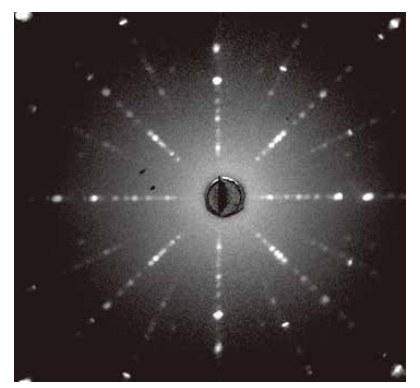

(e)
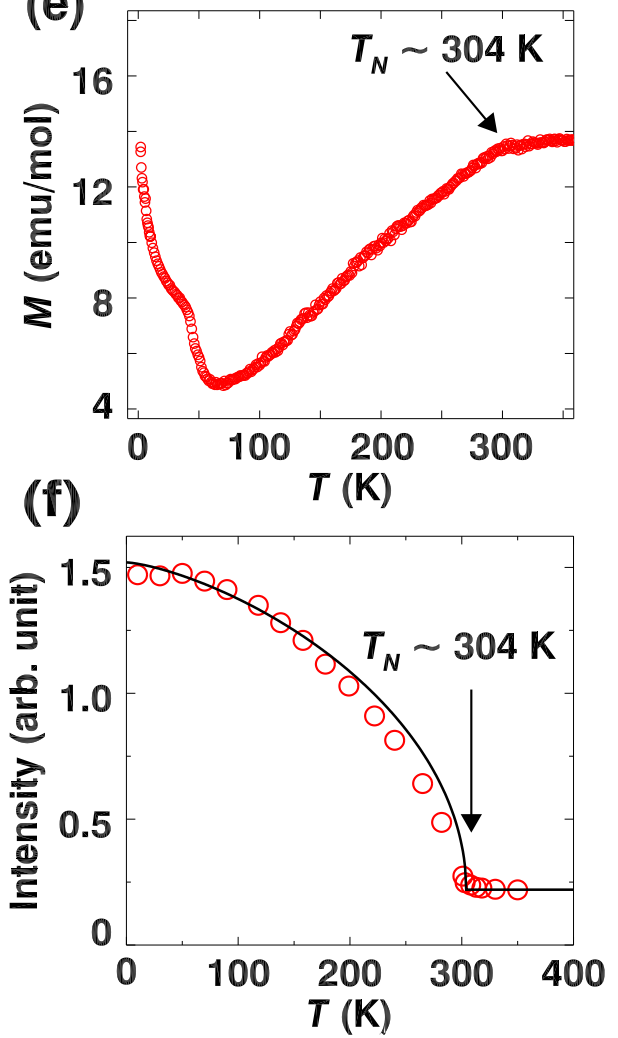

FIG. 1. (a) Crystal and magnetic structure of $\mathrm{Sr}_{1-x} \mathrm{Mn}_{1-y} \mathrm{Sb}_{2}$ (space group No. 62, Pnma) with $a \approx b=4.4342, c=$ $23.1359 \AA$, and $\alpha=\beta=\gamma=90^{\circ}$. Note that we only show half of the unit cell along the $c$ axis. Arrows represent the spins. The exchange paths are labeled with $J_{1}$ (nearest-neighbor), $J_{2}$ (next-nearest-neighbor), and $J_{c}$ (interlayer). (b) Coaligned single crystals we used for the inelastic neutron scattering measurements. (c) Single-crystal X-ray diffraction pattern of the cleavage plane. (d) Backscattering Laue x-ray diffraction pattern with the beam along the [001] direction. (e) Temperature dependence of the magnetization measured with magnetic field $B=1 \mathrm{~T}$ applied along the $c$ axis. (f) Temperature dependence of the integrated intensities of the magnetic Bragg peak $(1,0,0)$. The solid line in (f) is a guideline to the eye. Arrows in (e) and (f) indicate the Néel temperature $T_{\mathrm{N}}$.

dexed with $(0,0, L)$ as shown in Fig. 1.(c). The sharp $(0,0, L)$ peaks in Fig. 1(c) and clear Laue pattern in Fig. 1(d) demonstrate the excellent crystallinity of the single crystals. EDS measurements on the single crystals show that there are approximately $1 \% \mathrm{Sr}$ and $7 \%$ Mn deficiencies $(x<0.1, y<0.1)$, which is commonly seen in this materia $37 / 40$. In fact, the magnetic properties of $\mathrm{Sr}_{1-x} \mathrm{Mn}_{1-y} \mathrm{Sb}_{2}$ are influenced by the amount of deficiency. Samples with larger $\mathrm{Sr}$ and smaller $\mathrm{Mn}$ deficiencies display stronger ferromagnetic behavior, while those with larger Mn deficiency show weaker ferromagnetic behavior ${ }^{37}$. According to the magnitude of the ferromagnetic saturated moment $M_{s}$, Ref. 37 categorized the samples into three types; $M_{s} \sim 0.1-0.6 \mu_{B} / \mathrm{Mn}$ for type $\mathrm{A}, \sim 0.04-0.06 \mu_{B} / \mathrm{Mn}$ for type $\mathrm{B}$, and $\sim 0.004-0.006 \mu_{B} / \mathrm{Mn}$ for type C. Our samples with larger Mn deficiency and weaker ferromagnetic behavior fall into the category of type C. The temperature dependence of the magnetization is shown in Fig. 1(e). At the $T_{\mathrm{N}}$ of $\sim 304 \mathrm{~K}$, the magnetization starts to show a sup- pression due to the development of the antiferromagnetic order. This value is consistent with previous reports for this materia $37 / 40$. As shown in Fig. 1 (a), the antiferromagnetic order has a canted ferromagnetic moment along the $a$ axis 37 . At low temperatures, the ferromagnetic moment develops and gives rise to the magnetization upturn shown in Fig. 1(e). Such an upturn has also been observed in Ref. 37. The magnitude of the upturn corresponds to the strength of the ferromagnetic moment and depends on the stoichiometry, especially the amount of the Mn deficiency of the sample $\mathrm{e}^{37}$. Therefore, we suspect that the kink around $40 \mathrm{~K}$ in Fig. 1(e) is due to the presence of a small amount of second phase with a slightly different stoichiometry. In Fig. 11(f), we plot the temperature dependence of the integrated intensities of the magnetic Bragg peak $(1,0,0)$, from which we observe that the peak intensity has an onset at the $T_{\mathrm{N}}$ of $304 \mathrm{~K}$ upon cooling. These results are consistent with previous literatures on this material37/40. 


\section{B. Topological Electronic Properties}
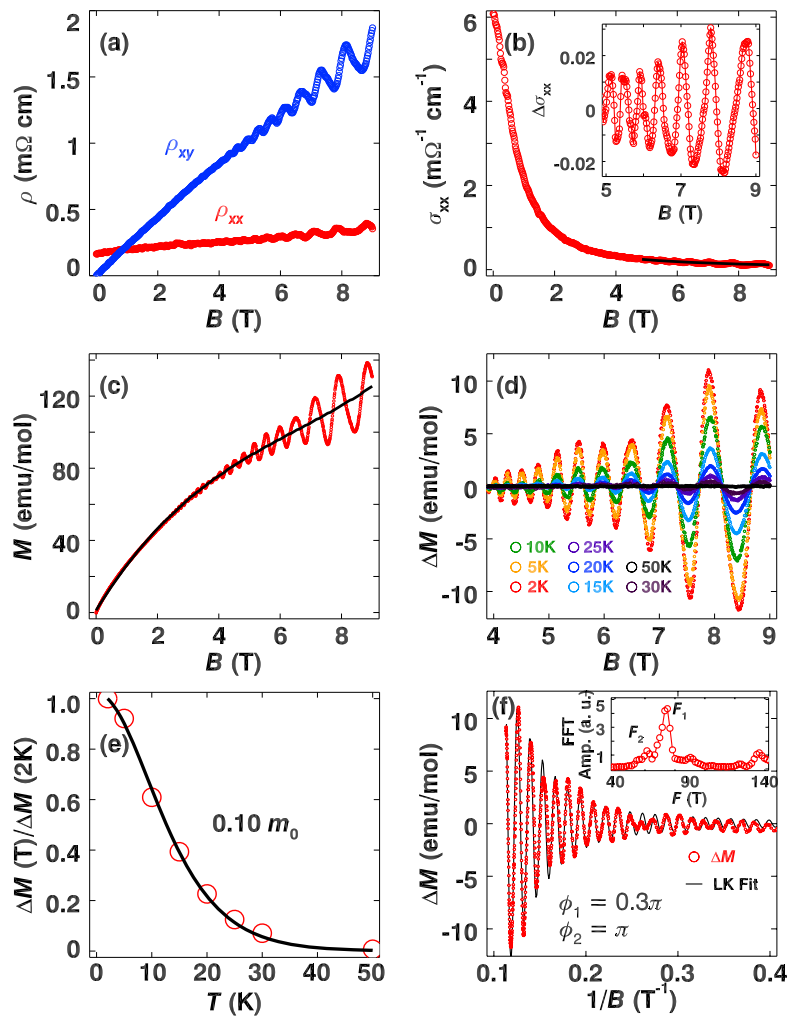

FIG. 2. (a) The in-plane longitudinal $\left(\rho_{x x}\right)$ and transverse (Hall) $\left(\rho_{x y}\right)$ resistivity as a function of magnetic field applied along the $c$ axis measured at $2 \mathrm{~K}$. (b) Magnetic-field dependence of the in-plane conductivity $\left(\sigma_{x x}\right)$. The solid line indicates the smooth background. The inset is the oscillatory component $\left(\Delta \sigma_{x x}\right)$ by subtracting the smooth background. (c) Magnetic-field dependence of the magnetization measured at $2 \mathrm{~K}$, with the solid line being the fit of the smooth background. (d) Oscillatory component of the magnetization $(\Delta M)$ by subtracting the background at various temperatures. (e) Temperature dependence of the oscillatory amplitude, normalized by $\Delta M$ at $2 \mathrm{~K}$. The solid line through data is the fit as described in the main text, from which we obtain an effective mass of $0.1 m_{0}$. (f) $\Delta M$ at $2 \mathrm{~K}$ plotted as a function of $1 / B$. The inset shows the fast Fourier transform spectra of $\Delta M$ which show two oscillating frequencies. The solid line is the fit to $\Delta M$ with a two-frequency LifshitzKosevich formula. From the fit, we obtain the Berry phase of $0.3 \pi$ and $\pi$ corresponding to the frequency of 73.2 and $57.2 \mathrm{~T}$, respectively.

We first probed the electronic properties of $\mathrm{Sr}_{1-x} \mathrm{Mn}_{1-y} \mathrm{Sb}_{2}$ by measuring the resistivity and magnetization under external magnetic field. In Fig. 2(a), we plot the in-plane longitudinal $\left(\rho_{x x}\right)$ and transverse (Hall) $\left(\rho_{x y}\right)$ resistivity measured at $2 \mathrm{~K}$ with field applied along the $c$ axis. In Fig. 2(b), we plot the in-plane conductivity $\sigma_{x x}$, which is converted from the $\rho_{x x}$ and $\rho_{x y}$, using $\sigma_{x x}=\rho_{x x} /\left(\rho_{x x}^{2}+\rho_{x y}^{2}\right)$. By subtracting the background, we obtain the oscillatory component $\Delta \sigma_{x x}$, as shown in the inset of Fig. 2(b). These strong $\mathrm{SdH}$ oscillations at moderate magnetic field suggest the high mobility and light effective mass of the carriers 46 . Furthermore, the large $\mathrm{SdH}$ oscillation amplitude is also an indication of the quasi-two-dimensional electronic structure of the materia 53 . In this study, we focus on the type-C samples. Compared to type-A and type-B samples which have larger saturated ferromagnetic moments 37 , type-C samples exhibit relatively weak quantum oscillations due to the large $\rho_{x y}$. Moreover, $\mathrm{SdH}$ oscillations originate from the oscillating scattering rate and the details depend on the actual scattering mechanisms. This sometimes makes the Lifshitz-Kosevich (LK) theory not accurate in describing experimental observations 5 , as is the case for $\mathrm{Sr}_{1-x} \mathrm{Mn}_{1-y} \mathrm{Sb}_{2}$ (Ref. 37). On the other hand, the dHvA effect is caused directly by the oscillations of electrons' free energy and can be well described by the LK model for both three- and two-dimensional systems ${ }^{53}$. Among the three types of samples, type C exhibits the strongest $\mathrm{dHvA}$ oscillations due to the weak ferromagnetic background 37 . As shown in Fig. 2(c), the magnetization shows very clear oscillations at moderate fields. Therefore, we use dHvA oscillations for detailed analyses of the electronic properties for our type-C samples below.

By subtracting the background of the magnetization, we obtain the oscillatory component of the magnetization $\Delta M$ as a function of magnetic field $B$ [Fig. 2(d)]. The oscillatory component can be described by the LK formula 53154 , as $\Delta M \propto-B^{-\lambda} R_{T} R_{D} R_{S} \sin [2 \pi(F / B-$ $\gamma+\delta)]$, where the exponent $\lambda$ is $1 / 2$ and 0 for the three- and two-dimensional cases, respectively; Onsager phase factor $\gamma=1 / 2-\phi / 2 \pi$, and $\delta$ is the phase shift, taking the value of $\pm 1 / 8$ and 0 for for threeand two-dimensional cases, respectively; $F$ and $\phi$ are the oscillation frequency and Berry phase, respectively; $R_{\mathrm{T}}=\left(2 \pi^{2} k_{\mathrm{B}} T / \hbar \omega_{c}\right) / \sinh \left(2 \pi^{2} k_{\mathrm{B}} T / \hbar \omega_{c}\right)$, and $R_{\mathrm{D}}=$ $\exp \left(-2 \pi^{2} k_{\mathrm{B}} T_{\mathrm{D}} / \hbar \omega_{c}\right)$, with $\omega_{c}$ and $T_{\mathrm{D}}$ being the cyclotron frequency and Dingle temperature, respectively. $R_{\mathrm{S}}=$ $\cos \left(\frac{1}{2} \pi g m^{*} / m_{0}\right)$ is the spin damping factor due to the Zeeman splitting, which can be neglected in the present case, as we do not observe any sign of Landau level splitting in the oscillations at $T=2 \mathrm{~K}$ under $B=9 \mathrm{~T}$. Here, $m^{*}$ and $m_{0}$ are the effective mass and rest mass of the free electrons respectively.

Since the cyclotron frequency $\omega_{c}=e B / m^{*}$, the only temperature-dependent term in $\Delta M$ can be written as,

$$
\begin{aligned}
R_{T} & =\left(2 \pi^{2} k_{B} T / \hbar \omega_{c}\right) / \sinh \left(2 \pi^{2} k_{B} T / \hbar \omega_{c}\right) \\
& \approx\left(14.69 m^{*} T / B\right) / \sinh \left(14.69 m^{*} T / B\right) .
\end{aligned}
$$

We have fitted the temperature dependence of the amplitude of $\Delta M$ using this formula, and the results are shown in Fig. 2(e). We obtain an effective mass of $0.10(3) m_{0}$ for the sample by averaging the results obtained at several fields. This value is similar to those of NbP (Ref. [55), $\mathrm{ZrSiS}$ (56), and $\mathrm{Cd}_{3} \mathrm{As}_{2}$ (8, 57, and (58). This effective mass is slightly different from that in Ref. 37, which may 
be caused by the composition difference and the limited magnetic field range in our study.

To obtain the Berry phase $\phi$ of $\mathrm{Sr}_{1-x} \mathrm{Mn}_{1-y} \mathrm{Sb}_{2}$, we can use the Onsager quantization rule to construct the Landau-level fan diagram 5960 . However, as observed in Fig. 2(d), there appear to be two oscillating frequencies. We perform fast Fourier transform analysis of the dHvA oscillation as shown in the inset of Fig. 2(f). This clearly shows two frequencies of $F_{1} \approx 73.2 \mathrm{~T}$ and $F_{2} \approx 57.2 \mathrm{~T}$, similar to the values for the type-C samples in Ref. 37. These values are in excellent agreement with the angle-resolved photoemission spectroscopy data and band structure calculations for this materia $\frac{35] 38}{2}$. We further analyze the dHvA data with a two-frequency LK model, and the results are shown in Fig. 2(f). From the LK fit, we obtain the Berry phase of $0.3 \pi$ and $\pi$ corresponding to the bands of 73.2 and $57.2 \mathrm{~T}$, respectively. These strongly indicate that the band structure is topologically nontrivial. We have measured the resistivity and magnetizations for other type-C samples. Although the details depend on the stoichiometry of the samples, all of them exhibit nontrivial topological properties with nonzero Berry phase and light effective mass. These results are consistent with the suggestion that type-A, -B, and -C samples are all topologically nontrivial despite of the quantitative difference among different sample types 37 .

\section{INS Spectra}

Having studied the topological electronic properties of the material, we now move to investigate the spin dynamics by INS measurements to explore whether and how topological fermions affect magnons. In Fig. 3(a) and (b), we present magnetic dispersions along two highsymmetry directions of [100] and [110], respectively, measured with an incident energy $E_{i}$ of $100.1 \mathrm{meV}$ at $6 \mathrm{~K}$. Along the [100] direction, the excitation spectra disperse from the antiferromagnetic ordering wavevector $(1,0,0)$ and reach the band top at about $76 \mathrm{meV}$ at $(0.5,0,0)$ and $(-1.5,0,0)$ [Fig. 3(a)]. Note that the $(1,0,0)$ peak is structurally forbidden 37 , and only appears below the antiferromagnetic transition temperature $T_{\mathrm{N}}$ of $304 \mathrm{~K}$ as shown in Fig. 1(f), so these excitations are not contributed by phonons but purely magnetic. The weak spectral weight for wave vectors $\boldsymbol{Q}_{\mathrm{s}}$ ranging from $(-0.5,0,0)$ to $(0.5,0,0)$ is due to the small magnetic structure factor. The signal at the $\Gamma$ point with $H=0$ is expected to be weak due to the antiferromagnetic structure ${ }^{37}$. The spin waves remain well defined up to about $70 \mathrm{meV}$. Along the [110] direction, the band top is reduced to about $50 \mathrm{meV}$, as shown in Fig. 3(b), indicating the strong anisotropy within the $a-b$ plane. The dispersion shows a local minimum at $(-0.5,0.5,0)$. To probe the low-energy excitations, we used an $E_{i}$ of $16 \mathrm{meV}$, and the obtained dispersion along the [100] direction is shown in Fig. 3(c). The dispersion in the left panel of Fig. 3(c) clearly shows a spin gap of $6 \mathrm{meV}$. The gap is also evident from the cut at (100) along the energy axis [right panel of Fig. 3(c)]. Although the spin-gap value is similar to that obtained for the type-A sample with strong ferromagnetic moment in Ref. 40, the high-energy behavior in Fig. 3(c) is different from that in Ref. 40, possibly because we use type-C samples, different from Ref. 40, In Fig. 3. (d), we plot the dispersion along the [001] direction. The out-of-plane dispersion also exhibits a clear spin gap at $6 \mathrm{meV}$, and the total band width is about $5 \mathrm{meV}$, an order of magnitude smaller than those in the plane shown in Fig. 3(a) and (b), consistent with the quasi-two-dimensional crystal structure of this material. The magnetic excitations with a spin gap of $6 \mathrm{meV}$, highly dispersive in the plane, but weakly dispersive out of the plane, are qualitatively similar to those in related magnetic Dirac materials such as $\mathrm{CaMnBi}_{2}{ }_{21}^{21}, \mathrm{SrMnBi}_{2} 21$, and $\mathrm{YbMnBi}_{2}{ }_{34 \mid 51}$. These spinwave excitations remain well defined at high energies, distinct from some iron-based superconductors where the high-energy excitations are strongly damped and the spin waves decay into a Stoner continuum $61 \mid 62$.

To visualize the magnetic excitations in the momentum space, we plot a series of constant-energy contours in the ( $H K 0)$ plane with energies up to $70 \mathrm{meV}$ in Fig. 4. At an energy transfer of $\Delta E=10 \pm 2 \mathrm{meV}$, slightly above the spin gap of $6 \mathrm{meV}$, there are both magnetic and lattice excitations. For instance, in Fig. 4(a), there is a strong peak at $(-1,0,0)$, which corresponds to a magnetic Bragg peak at the elastic position. On the other hand, the peaks at ($1, \pm 1,0)$ are phonons, which disappear at higher energies, as the phonon intensities decrease rapidly as the energy increases. The spin waves disperse outward as the energy increases and form a ring centering $(-1,0,0)$ as shown in Fig. 4(b); upon further increasing the energy to $50 \mathrm{meV}$, the ring evolves into a square. At $70 \mathrm{meV}$, slightly below the band top, there are still well-resolved magnetic excitations. The clear patterns of the spin waves allow us to obtain an accurate parameterization of the spectra, as we discuss below.

To analyze the data, we use the following Hamiltonian21|34|51

$$
\hat{H}=\sum_{<i, j>} J_{i j} \hat{S}_{i} \cdot \hat{S}_{j}-\sum_{i} D\left(\hat{S_{i}^{Z}}\right)^{2}
$$

where we include a nearest-neighbor $\left(J_{1}\right)$ and nextnearest-neighbor $\left(J_{2}\right)$, and interlayer exchange coupling constant $\left(J_{c}\right)$. The exchange paths are illustrated in Fig. 1(a). To account for the spin gap, we include a single-ion anisotropy term $D$. Based on the linear spinwave theory and SPINW package $e^{63}$, we calculated the spin-spin correlation function $\frac{6465}{}$,

$$
S^{\alpha \beta}(\boldsymbol{Q}, \omega)=\frac{1}{N} \sum_{i j} \mathrm{e}^{\mathrm{i} \boldsymbol{Q}\left(\boldsymbol{r}_{\boldsymbol{i}}-\boldsymbol{r}_{j}\right)} \int_{-\infty}^{\infty}\left\langle S_{i}^{\alpha} S_{j}^{\beta}(t)\right\rangle \mathrm{e}^{-\mathrm{i} \omega t} d t,
$$

which is related to scattering cross section

$$
\frac{d^{2} \sigma}{d \Omega d \omega} \propto|F(\boldsymbol{Q})|^{2} \sum_{\alpha \beta}\left(\delta_{\alpha \beta}-\frac{Q_{\alpha} Q_{\beta}}{Q^{2}}\right) S^{\alpha \beta}(\boldsymbol{Q}, \omega),
$$



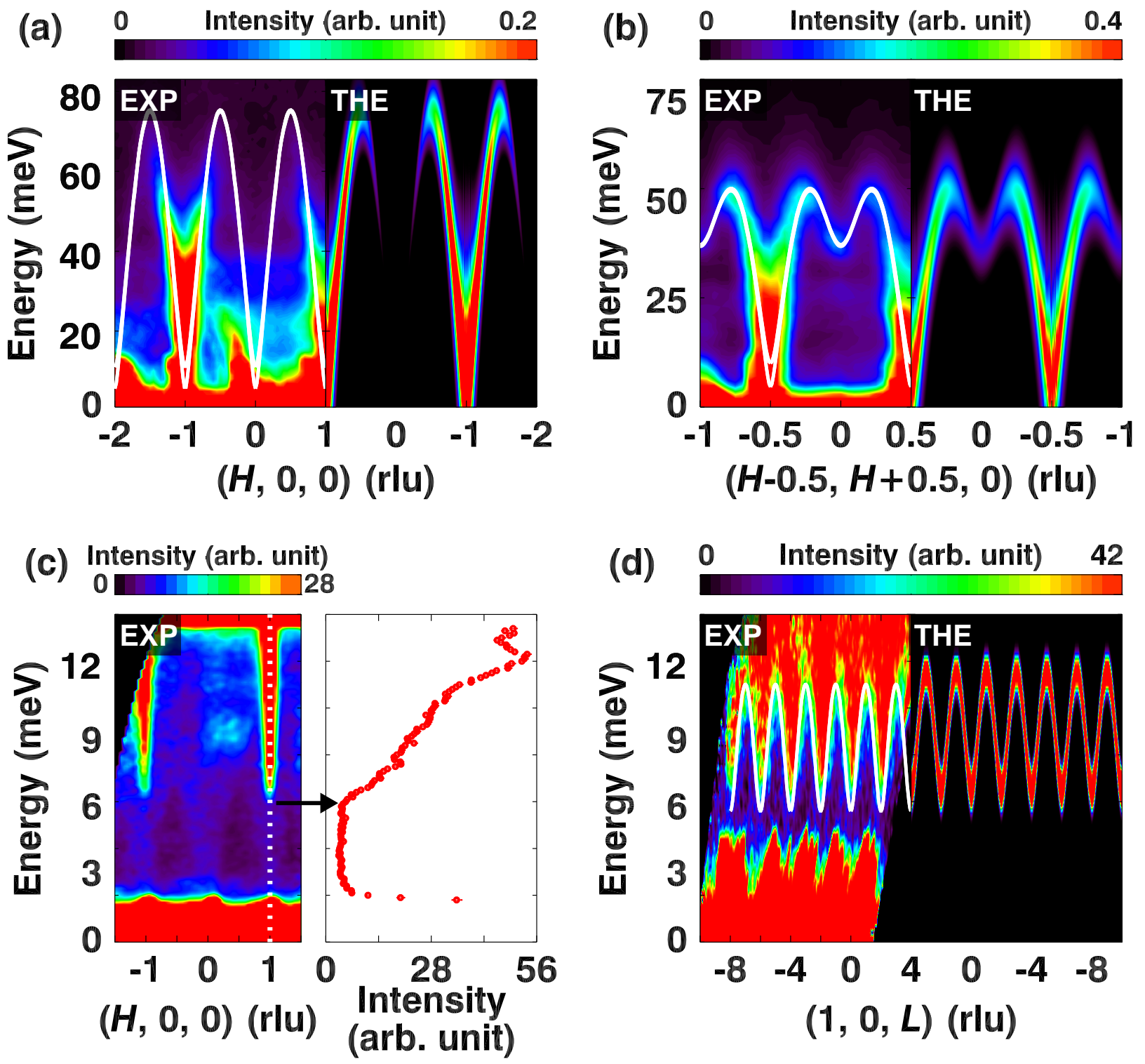

FIG. 3. Magnetic dispersions of $\mathrm{Sr}_{1-x} \mathrm{Mn}_{1-y} \mathrm{Sb}_{2}$ measured at $6 \mathrm{~K}$ together with the theoretical calculation results. (a) and (b) Dispersions along the [100] and [110] directions, respectively, obtained on 4SEASONS with $E_{i}=100.1 \mathrm{meV}$. (c) and (d) Dispersions along the [100] and [001] directions obtained on MERLIN with $E_{i}=16 \mathrm{meV}$. Panel (c) focuses on the low-energy part of (a) to show the spin gap. In panels (a), (b), and (d), solid lines are the calculated dispersions. The calculated dispersions with intensities are shown on the right hand side of each panel to be compared with the experimental data on the left hand side. Data in (a) and (c) were integrated with $K=[-0.2,0.2] \mathrm{rlu}$, and all available $L \mathrm{~s}$; data in (b) with a thickness of 0.1 rlu about each momentum along the [-110] direction; data in (d) with $H=[0.8,1.2]$ rlu, and $K=[-0.2,0.2]$ rlu. The dashed line in (c) illustrates an energy scan at (100). The scan profile is plotted on the right hand side with the energy and intensity being the $y$ and $x$ axis, respectively. The arrow indicates the gap value. In (d), the low-energy part below about 4.5 meV is due to the Bragg peaks and some spurious signals, and the broad experimental data can be reproduced by spin-wave calculations convoluting the instrumental resolution 21 .

where $\alpha$ and $\beta$ are direction indices $(x y z), F(\boldsymbol{Q})$ is the magnetic structure factor, $\boldsymbol{S}_{i}$ is the spin at site $i$ with the coordinate $\boldsymbol{r}_{i}$, and $N$ is the total number of sites. In Figs 3 and 4 , the calculated spectra using $S J_{1} \sim 28.0$, $S J_{2} \sim 9.3, S J_{c} \sim-0.1$, and $S D \sim-0.07 \mathrm{meV}$ are plotted on the right hand side of the experimental results in Fig. 3(a), (b), and (d). Furthermore, the calculated dispersions are plotted as solid lines on top of the experimental dispersions. It is clear that the calculations are in excellent agreement with the experimental data, including the dispersions in and out of plane, the spin anisotropy gap, and the spectral weight distribution in 
(a)

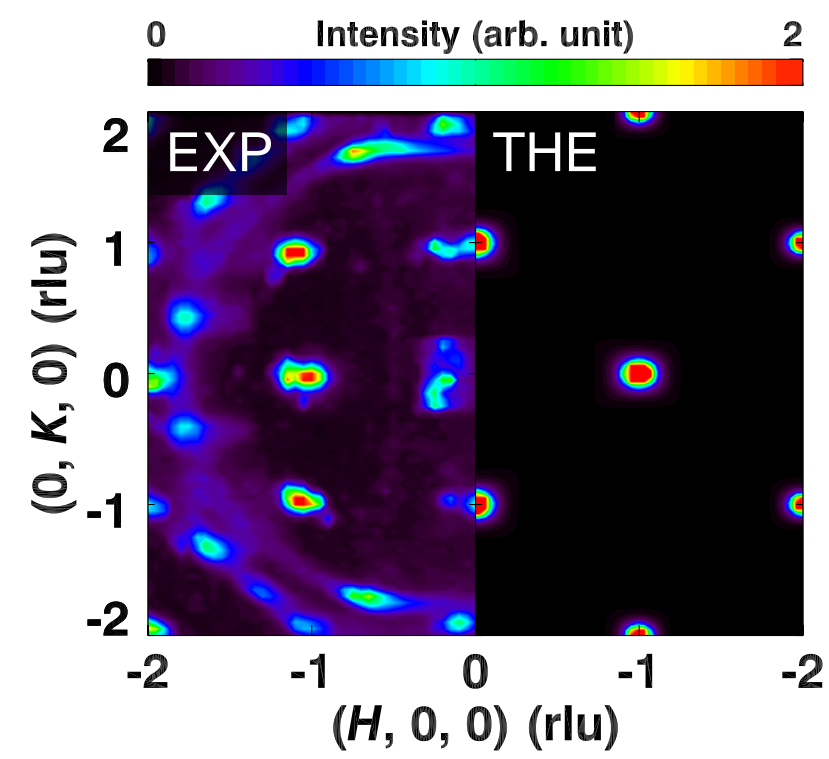

(c)

$$
\Delta E=[48,52] \mathrm{meV}
$$

0 Intensity (arb. unit)

0.15

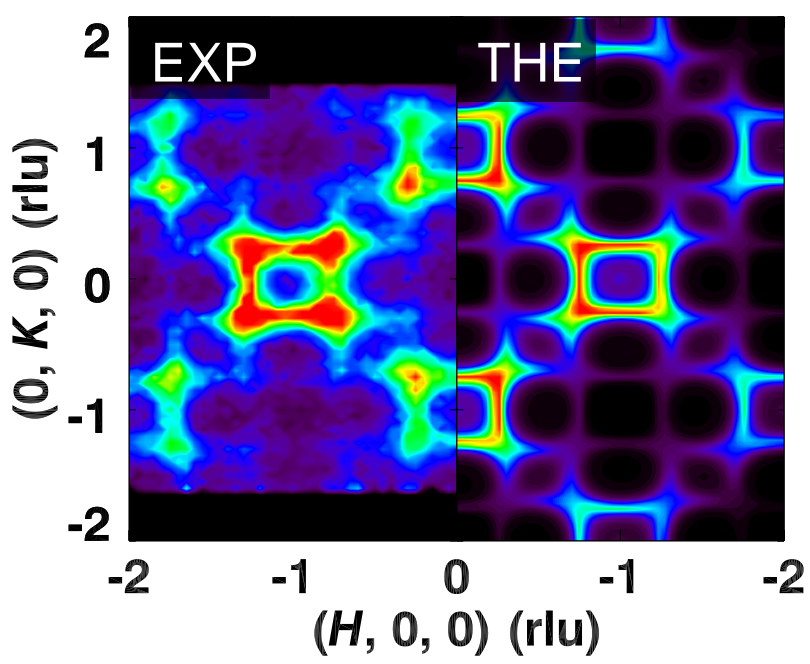

(b) $\Delta E=[28,32] \mathrm{meV}$

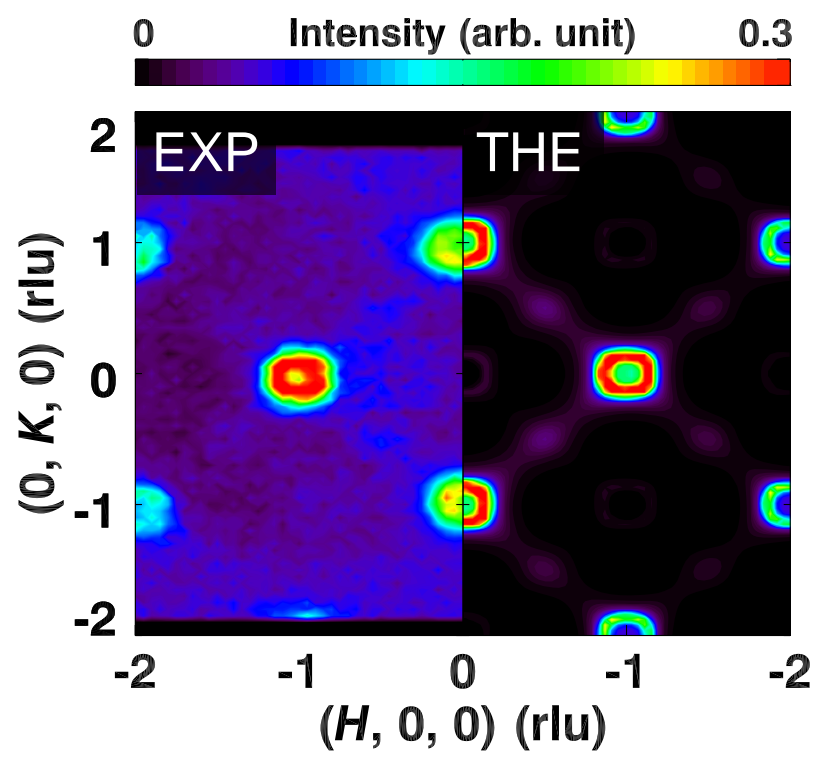

(d)

$$
\Delta E=[68,72] \mathrm{meV}
$$
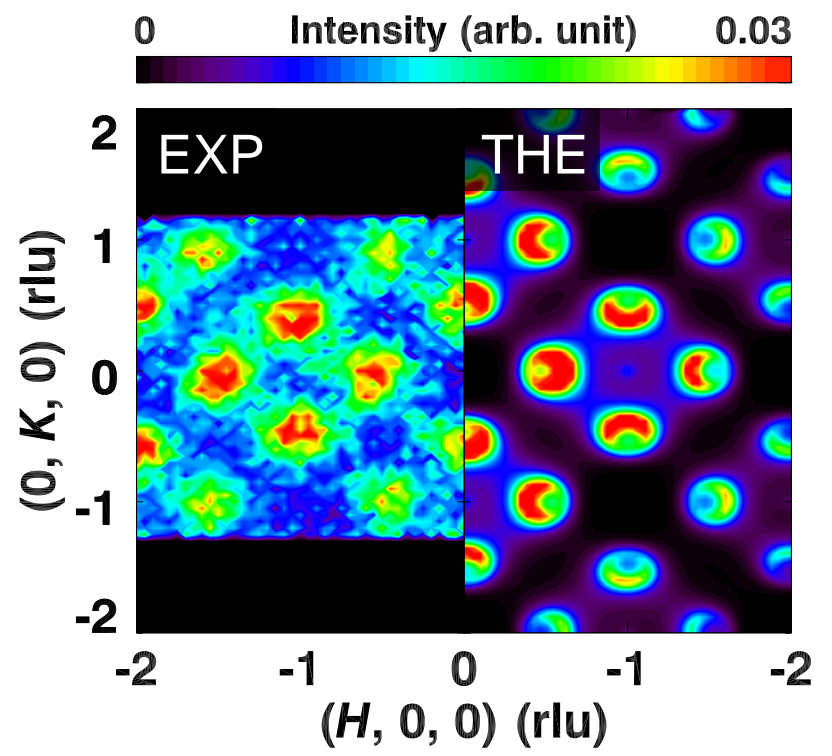

FIG. 4. Contour maps of the constant-energy cuts measured with $E_{i}=100.1 \mathrm{meV}$ at $6 \mathrm{~K}$. The experimental data are shown on the left hand side of each panel, while the theoretical results are shown on the right hand side. The energy range of the integration is shown on the top of each panel. All the data are from 4SEASONS.

the momentum space. The parameters we used in these calculations, and the gap as well as the $T_{\mathrm{N}}$ are tabulated

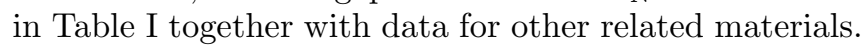

There are a few remarks we want to make about the calculations and fittings. First, since there are four Mn atoms in each unit cell 37 , there are four spin-wave branches in the calculations as shown in Fig. 3(a) and (b). However, due to the resolution limit, we are not able to resolve these four branches experimentally. Sec- ond, the structure factor is small for $\boldsymbol{Q}$ ranging from $(-0.5,0,0)$ to $(0.5,0,0)$, consistent with the experimental observations of vanishing scattering intensities in this range. Third, a positive $J_{2}$ is needed to account for the local minimum at $(-0.5,0.5,0)$, as shown in Fig. 3(b). The perfect match of the calculated dispersion and the experimental spectra further demonstrates the quality of the fits. Without such a $J_{2}$, the local minimum is absent, as shown in Ref. 40. The same sign of $J_{1}$ and $J_{2}$ intro- 
TABLE I. Exchange parameters, single-ion anisotropy constants, spin gaps, ordering temperature $T_{N}$, and related references. Values in the brackets denote the errorss.

\begin{tabular}{|c|c|c|c|c|c|c|c|}
\hline & $S J_{1}(\mathrm{meV})$ & $S J_{2}(\mathrm{meV})$ & $S J_{C}(\mathrm{meV})$ & $S D(\mathrm{meV})$ & $\Delta(\mathrm{meV})$ & $T_{N}(\mathrm{~K})$ & References \\
\hline $\mathrm{Sr}_{1-x} \mathrm{Mn}_{1-y} \mathrm{Sb}_{2}$ & $28.0(2)$ & $9.27(36)$ & $-0.10(1)$ & $-0.07(8)$ & $\sim 6.0$ & $\sim 304$ & our work \\
\hline $\mathrm{Sr}_{1-x} \mathrm{Mn}_{1-y} \mathrm{Sb}_{2}$ & $23.25(75)$ & negligible & $-0.65(20)$ & $\operatorname{diag}(0.30(13) 0.8(3) 0)^{*}$ & $\sim 8.5$ & $\sim 304$ & 40 \\
\hline $\mathrm{SrMnBi}_{2}$ & $21.3(2)$ & $6.39(15)$ & $0.11(2)$ & $0.31(2)$ & $10.2(2)$ & $287(5)$ & 21 \\
\hline $\mathrm{CaMnBi}_{2}$ & $23.4(2)$ & $7.9(5)$ & $-0.10(5)$ & $0.18(3)$ & $8.3(8)$ & $264(2)$ & 21 \\
\hline $\mathrm{YbMnBi}_{2}$ & $25.2(2)$ & $10.1(3)$ & $0.131(4)$ & $-0.20(1)$ & $9.0(2)$ & $\sim 290$ & 34 and 51 \\
\hline
\end{tabular}

*In Ref. 40, $S^{\alpha} A_{i}^{\alpha \beta} S^{\beta}$ was used to replace the $S D$ in the Hamiltonian.

duces frustration into the system, as is also the case for $\mathrm{CaMnBi}_{2} 21, \mathrm{SrMnBi}_{2} \frac{21}{21}$, and $\mathrm{YbMnBi}_{2} 2$, as tabulated in TableI]. Fourth, the spin gap can be well reproduced by including the single-ion anisotropy term. In Ref. [50, by fitting the two-magnon Raman spectra in $\mathrm{CaMnBi}_{2}$ and $\mathrm{SrMnBi}_{2}$, an anomalously large interlayer coupling $J_{c}$ was obtained for both compounds and interpreted as due to the Bi Dirac band structure. In that work, the singleion anisotropy term was neglected. In our study, we find that the $J_{c}$ is fairly small $\left(S J_{c} \sim-0.10 \mathrm{meV}\right)$, similar to other INS work on related materials ${ }^{21 / 34}$. Such a small $J_{c}$ is consistent with the quasi-two-dimensional structure of these materials. Fifth, by comparing the experimental and theoretical results in Fig. 4(a), it is clear that the spin waves are well separated from the phonons as they emerge from different Bragg peaks. This makes analyzing the spin waves more easily. Sixth, the calculations of the dispersion along the [001] direction are much sharper than the experimental data, as shown in Fig. 3(d). Similar behavior was also observed in Ref. 21, and it was shown that by convoluting the instrumental resolution in the calculations, the broad experimental spectra could be reproduced.

\section{DISCUSSIONS AND CONCLUSIONS}

From the electrical transport and magnetization results, we find that $\mathrm{Sr}_{1-x} \mathrm{Mn}_{1-y} \mathrm{Sb}_{2}$ hosts topological fermions, consistent with the proposal of magnetic Weyl

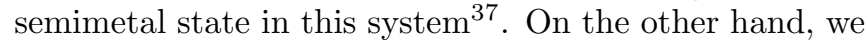
observe well-defined spin waves resulting from the Mn atoms, with no discernible damping due to the itinerant electrons. The spectra can be well fitted by a localmoment Heisenberg model. From these data, we do not detect any effect of the itinerant electrons on the spinwave excitations resulting from the Mn local moments. Similarly, in $\mathrm{CaMnBi}_{2}{ }^{21}, \mathrm{SrMnBi}_{2} 21$, and $\mathrm{YbMnBi}_{2} \frac{34}{\text {, }}$ no influence of the Dirac fermions on the spin dynamics has been observed either. These behaviors are different from those in iron-based superconductors where itinerant electrons are believed to play an important role in damping the high-energy magnetic excitations and making the spin waves decay into a Stoner continuum 6162 .

In $\mathrm{Sr}_{1-x} \mathrm{Mn}_{1-y} \mathrm{Sb}_{2}$, the magnetic excitations are resulting from the local moments in the Mn layers, whereas the itinerant electrons are contributed by the Sb layers which are separated from the former $13 / 21 / 34|35| 38$. The material has a quasi-two-dimensional structure with a large $c$-axis constant of $23.1359 \AA$. The large SdH oscillation amplitude also indicates that the electronic structure for $\mathrm{Sr}_{1-x} \mathrm{Mn}_{1-y} \mathrm{Sb}_{2}$ is two-dimensional like. Furthermore, the spin-wave spectra indicate that the magnons are quasi-two-dimensional as well. Our fitting indicates that $S J_{1}$ and $S J_{2}$ are 28.0 and $9.27 \mathrm{meV}$, respectively, while $J_{c}$ is only $-0.10 \mathrm{meV}$, two orders of magnitude smaller. Taking these into account, it is not surprising that we do not observe the effect of the itinerant electrons on the spin dynamics. We believe this also explains the similar behavior in other magnetic Dirac semimetal systems 21/34. Nevertheless, our results do not completely rule out the possible subtle influence of the itinerant electrons on the magnons.

Baring these in mind, in order to observe strong spinfermion coupling, it may be helpful to look for quasitwo-dimensional magnetic Dirac or Weyl semimetals with stronger interlayer couplings, or even three-dimensional materials. A more ideal case will be that the magnons and fermions are in the same layer or arising from the same atoms like in iron-based superconductors $61 \mid 62$. The recently discovered magnetic Weyl semimetal $\mathrm{Co}_{3} \mathrm{Sn}_{2} \mathrm{~S}_{2}$ may be such an example ${ }^{6667}$. In this material, it was shown that the Weyl nodes were close to the Fermi leve 67/68. Furthermore, it exhibited both high anomalous Hall effect and large anomalous Hall angle $69 / 70$, indicating strong coupling between the magnetism and electronic transport. It would be of great interest to further explore the possible spin-fermion coupling in this and other related materials 71 .

Likewise, to investigate the spin-fermion interactions, one can also make discussions in the context of how the electronic structures are affected by the magnetism. For example, in a magnetic Weyl semimetal $\mathrm{Mn}_{3} \mathrm{Sn}$, it was suggested that the helical magnetic ordering observed in the INS measurements could remove the Weyl nodes in the helical phase ${ }^{72}$. Similar practice had also been taken in $\mathrm{Sr}_{1-x} \mathrm{Mn}_{1-y} \mathrm{Sb}_{2}$, where the authors extracted the exchange parameters from the spin excitation spectra obtained from INS measurements, and then with the aid of density functional theory, they suggested that the magnetic order could tune the topological band structure 40 . However, it is not clear how the inclusion of a substantial $J_{2}$, as shown in our current work and typically present in these materials (Table I), will affect the results. It will 
be beneficial to carry out angle-resolved photoemission spectroscopy measurements to test these proposals.

To conclude, while our electrical transport and magnetization measurements indicate that $\mathrm{Sr}_{1-x} \mathrm{Mn}_{1-y} \mathrm{Sb}_{2}$ is a topological semimetal, our INS data reveal well-defined spin waves resulting from the Mn local moments, which can be nicely described using a Hamiltonian including $S J_{1} \sim 28.0 \mathrm{meV}, S J_{2} \sim 9.3 \mathrm{meV}, S J_{c} \sim-0.1 \mathrm{meV}$, and $S D \sim-0.07 \mathrm{meV}$. Our results suggest that the topological fermions do not impact the magnons. We propose this to be due to the separated layers of the localized spins and itinerant carriers and the weak interlayer coupling.

\section{ACKNOWLEDGEMENTS}

We would like to thank Yuefeng Nie for providing access to the single-crystal X-ray diffractometer. We are grateful for the stimulating discussions with Fucong Fei. The work was supported by the National Natural Science Foundation of China with Grant Nos 11822405, $11674157,11674158,11774152$, and 11904170, National Key Projects for Research and Development of China with Grant No. 2016YFA0300401, Natural Science Foundation of Jiangsu Province with Grant Nos BK20180006 and BK20190436, Fundamental Research Funds for the Central Universities with Grant No. 020414380117, and the Office of International Cooperation and Exchanges of Nanjing University. The experiment at the Materials and Life Science Experimental Facility of the JPARC was performed under a user program (Proposal No. 2018A0018). We also acknowledge the financial support by the Newton Fund for China from ISIS Facility of Rutherford Appleton Laboratory.
* These authors contributed equally to the work.

† slyu@nju.edu.cn

‡ jxli@nju.edu.cn

$\S$ jwen@nju.edu.cn

1 N. P. Armitage, E. J. Mele, and Ashvin Vishwanath, "Weyl and Dirac semimetals in three-dimensional solids," Rev. Mod. Phys. 90, 015001 (2018).

2 T. O. Wehling, Annica M Black-Schaffer, and Alexander V Balatsky, "Dirac materials," Adv. Phys. 63, 1-76 (2014).

3 Oskar Vafek and Ashvin Vishwanath, "Dirac fermions in solids: From high- $\mathrm{T}_{c}$ cuprates and graphene to topological insulators and Weyl semimetals," Annu. Rev. Condens. Matter Phys. 5, 83-112 (2014)

${ }^{4}$ A. H. Castro Neto, F. Guinea, N. M. R. Peres, K. S. Novoselov, and A. K. Geim, "The electronic properties of graphene," Rev. Mod. Phys. 81, 109-162 (2009).

5 Tian Liang, Quinn Gibson, Mazhar N. Ali, Minhao Liu, R. J. Cava, and N. P. Ong, "Ultrahigh mobility and giant magnetoresistance in the Dirac semimetal $\mathrm{Cd}_{3} \mathrm{As}_{2}$," Nat. Mater. 14, 280-284 (2015)

${ }^{6}$ Chandra Shekhar, Ajaya K. Nayak, Yan Sun, Marcus Schmidt, Michael Nicklas, Inge Leermakers, Uli Zeitler, Yurii Skourski, Jochen Wosnitza, Zhongkai Liu, Yulin Chen, Walter Schnelle, Horst Borrmann, Yuri Grin, Claudia Felser, and Binghai Yan, "Extremely large magnetoresistance and ultrahigh mobility in the topological Weyl semimetal candidate NbP," Nat. Phys. 11, 645-649 (2015)

7 Yuanbo Zhang, Yan-Wen Tan, Horst L. Stormer, and Philip Kim, "Experimental observation of the quantum Hall effect and Berry's phase in graphene," Nature 438, 201-204 (2005)

${ }^{\delta}$ L. P. He, X. C. Hong, J. K. Dong, J. Pan, Z. Zhang, J. Zhang, and S. Y. Li, "Quantum transport evidence for the three-dimensional Dirac semimetal phase in $\mathrm{Cd}_{3} \mathrm{As}_{2}$," Phys. Rev. Lett. 113, 246402 (2014)

9 Shichao Li, Zhaopeng Guo, Dongzhi Fu, Xing-Chen Pan, Jinghui Wang, Kejing Ran, Song Bao, Zhen Ma, Zhengwei
Cai, Rui Wang, Rui Yu, Jian Sun, Fengqi Song, and Jinsheng Wen, "Evidence for a Dirac nodal-line semimetal in $\mathrm{SrAs}_{3}$," Sci. Bull. 63, 535 - 541 (2018)

10 Jun Xiong, Satya K. Kushwaha, Tian Liang, Jason W. Krizan, Max Hirschberger, Wudi Wang, R. J. Cava, and N. P. Ong, "Evidence for the chiral anomaly in the Dirac semimetal $\mathrm{Na}_{3} \mathrm{Bi}$," Science 350, 413-416 (2015).

11 Qiang Li, Dmitri E. Kharzeev, Cheng Zhang, Yuan Huang, I. PletikosiFan, A. Ting V. Fedorov, R. Ting D. Zhong, J. Ting A. Schneeloch, G. Ting D. Gu, and T. Valla, "Chiral magnetic effect in $\mathrm{ZrTe}_{5}, "$ Nat. Phys. 12, 550-554 (2016)

12 Sebastian Klemenz, Shiming Lei, and Leslie M. Schoop, "Topological semimetals in square-net materials," Annu. Rev. Mater. Res 49, 185-206 (2019)

is Joonbum Park, G. Lee, F. Wolff-Fabris, Y. Y. Koh, M. J. Eom, Y. K. Kim, M. A. Farhan, Y. J. Jo, C. Kim, J. H. Shim, and J. S. Kim, "Anisotropic Dirac fermions in a Bi square net of $\mathrm{SrMnBi}_{2}$," Phys. Rev. Lett. 107, 126402 (2011)

14 Jiakui K. Wang, Liang L. Zhao, Quan Yin, G. Kotliar, M. S. Kim, M. C. Aronson, and E. Morosan, "Layered transition-metal pnictide $\mathrm{SrMnBi}_{2}$ with metallic blocking layer," Phys. Rev. B 84, 064428 (2011)

15 Kefeng Wang, D. Graf, Hechang Lei, S. W. Tozer, and C. Petrovic, "Quantum transport of two-dimensional Dirac fermions in $\mathrm{SrMnBi}_{2}$," Phys. Rev. B 84, 220401 (2011).

16 Geunsik Lee, Muhammad A. Farhan, Jun Sung Kim, and Ji Hoon Shim, "Anisotropic Dirac electronic structures of $\mathrm{AMnBi}_{2}$ (A = Sr,Ca)," Phys. Rev. B 87, 245104 (2013).

17 Ya Feng, Zhijun Wang, Chaoyu Chen, Youguo Shi, Zhuojin Xie, Hemian Yi, Aiji Liang, Shaolong He, Junfeng He, Yingying Peng, Xu Liu, Yan Liu, Lin Zhao, Guodong Liu, Xiaoli Dong, Jun Zhang, Chuangtian Chen, Zuyan Xu, Xi Dai, Zhong Fang, and X. J. Zhou, "Strong anisotropy of Dirac cones in $\mathrm{SrMnBi}_{2}$ and $\mathrm{CaMnBi}_{2}$ revealed by angleresolved photoemission spectroscopy," Sci. Rep. 4, 5385- 
5385 (2014).

${ }^{18}$ L.-L. Jia, Z.-H. Liu, Y.-P. Cai, T. Qian, X.-P. Wang, H. Miao, P. Richard, Y.-G. Zhao, Y. Li, D.-M. Wang, J.-B. He, M. Shi, G.-F. Chen, H. Ding, and S.-C. Wang, "Observation of well-defined quasiparticles at a wide energy range in a quasi-two-dimensional system," Phys. Rev. B 90, $035133(2014)$

19 Y. J. Jo, Joonbum Park, G. Lee, Man Jin Eom, E. S. Choi, Ji Hoon Shim, W. Kang, and Jun Sung Kim, "Valley-polarized interlayer conduction of anisotropic Dirac fermions in $\mathrm{SrMnBi}_{2}$," Phys. Rev. Lett. 113, 156602 (2014)

20 Y. F. Guo, A. J. Princep, X. Zhang, P. Manuel, D. Khalyavin, I. I. Mazin, Y. G. Shi, and A. T. Boothroyd, "Coupling of magnetic order to planar Bi electrons in the anisotropic Dirac metals $\mathrm{AMnBi}_{2}(\mathrm{~A}=\mathrm{Sr}, \mathrm{Ca})$," Phys. Rev. B 90, 075120 (2014)

${ }_{21}$ M. C. Rahn, A. J. Princep, A. Piovano, J. Kulda, Y. F. Guo, Y. G. Shi, and A. T. Boothroyd, "Spin dynamics in the antiferromagnetic phases of the Dirac metals $\mathrm{AMnBi}_{2}$ (A = Sr, Ca)," Phys. Rev. B 95, 134405 (2017)

${ }^{22}$ Kefeng Wang, D. Graf, Limin Wang, Hechang Lei, S. W. Tozer, and C. Petrovic, "Two-dimensional Dirac fermions and quantum magnetoresistance in $\mathrm{CaMnBi}_{2}, "$ Phys. Rev. B 85, 041101 (2012)

${ }^{23}$ J. B. He, D. M. Wang, and G. F. Chen, "Giant magnetoresistance in layered manganese pnictide $\mathrm{CaMnBi}_{2}$," Appl. Phys. Lett. 100, 112405 (2012)

${ }^{24}$ Lijun Li, Kefeng Wang, D. Graf, Limin Wang, Aifeng Wang, and C. Petrovic, "Electron-hole asymmetry, Dirac fermions, and quantum magnetoresistance in $\mathrm{BaMnBi}_{2}$," Phys. Rev. B 93, 115141 (2016).

25 Yi-Yan Wang, Qiao-He Yu, and Tian-Long Xia, "Large linear magnetoresistance in a new Dirac material BaMnBi, ," Chin. Phys. B 25, 107503 (2016).

26 Sergey Borisenko, Daniil Evtushinsky, Quinn Gibson, Alexander Yaresko, Klaus Koepernik, Timur Kim, Mazhar Ali, Jeroen van den Brink, Moritz Hoesch, Alexander Fedorov, Erik Haubold, Yevhen Kushnirenko, Ivan Soldatov, Rudolf Schäfer, and Robert J. Cava, "Time-reversal symmetry breaking type-II Weyl state in $\mathrm{YbMnBi}_{2}$," Nat. Commun. 10, 3424 (2019).

27 Aifeng Wang, I. Zaliznyak, Weijun Ren, Lijun Wu, D. Graf, V. O. Garlea, J. B. Warren, E. Bozin, Yimei Zhu, and C. Petrovic, "Magnetotransport study of Dirac fermions in $\mathrm{YbMnBi}_{2}$ antiferromagnet," Phys. Rev. B 94, 165161 (2016)

${ }^{28}$ J. Y. Liu, J. Hu, D. Graf, T. Zou, M. Zhu, Y. Shi, S. Che, S. M. A. Radmanesh, C. N. Lau, L. Spinu, H. B. Cao, X. Ke, and Z. Q. Mao, "Unusual interlayer quantum transport behavior caused by the zeroth Landau level in $\mathrm{YbMnBi}_{2}, "$ Nat. Commun. 8, 646 (2017)

29 M. Chinotti, A. Pal, W. J. Ren, C. Petrovic, and L. Degiorgi, "Electrodynamic response of the type-II Weyl semimetal YbMnBi 2 ," Phys. Rev. B 94, 245101 (2016)

30 Dipanjan Chaudhuri, Bing Cheng, Alexander Yaresko, Quinn D. Gibson, R. J. Cava, and N. P. Armitage, "Optical investigation of the strong spin-orbit-coupled magnetic semimetal YbMnBi 2 ," Phys. Rev. B 96, 075151 (2017)

31 Andrew F. May, Michael A. McGuire, and Brian C. Sales, "Effect of Eu magnetism on the electronic properties of the candidate Dirac material EuMnBi 2 ," Phys. Rev. B 90, 075109 (2014).
32 Hidetoshi Masuda, Hideaki Sakai, Masashi Tokunaga, Yuichi Yamasaki, Atsushi Miyake, Junichi Shiogai, Shintaro Nakamura, Satoshi Awaji, Atsushi Tsukazaki, Hironori Nakao, Youichi Murakami, Taka-hisa Arima, Yoshinori Tokura, and Shintaro Ishiwata, "Quantum Hall effect in a bulk antiferromagnet $\mathrm{EuMnBi}_{2}$ with magnetically confined two-dimensional Dirac fermions," Sci. Adv. 2, e1501117 (2016)

$3 s$ H. Masuda, H. Sakai, M. Tokunaga, M. Ochi, H. Takahashi, K. Akiba, A. Miyake, K. Kuroki, Y. Tokura, and S. Ishiwata, "Impact of antiferromagnetic order on Landau-level splitting of quasi-two-dimensional Dirac fermions in EuMnBi 2 ," Phys. Rev. B 98, 161108 (2018).

34 Jian-Rui Soh, Henrik Jacobsen, Bachir Ouladdiaf, Alexandre Ivanov, Andrea Piovano, Tim Tejsner, Zili Feng, Hongyuan Wang, Hao Su, Yanfeng Guo, Youguo Shi, and Andrew T. Boothroyd, "Magnetic structure and excitations of the topological semimetal $\mathrm{YbMnBi}_{2}$," Phys. Rev. B 100, 144431 (2019)

${ }^{35}$ M Arshad Farhan, Geunsik Lee, and Ji Hoon Shim, "AeMnSb $2(\mathrm{Ae}=\mathrm{Sr}, \mathrm{Ba})$ : A new class of Dirac materials," J. Phys.: Condens. Matter 26, 042201 (2014).

36 J. B. He, Y. Fu, L. X. Zhao, H. Liang, D. Chen, Y. M. Leng, X. M. Wang, J. Li, S. Zhang, M. Q. Xue, C. H. Li, P. Zhang, Z. A. Ren, and G. F. Chen, "Quasi-twodimensional massless Dirac fermions in $\mathrm{CaMnSb}_{2}$," Phys. Rev. B 95, 045128 (2017).

J' J. Y. Liu, J. Hu, Q. Zhang, D. Graf, H. B. Cao, S. M. A. Radmanesh, D. J. Adams, Y. L. Zhu, G. Ting F. Cheng, X. Liu, W. A. Phelan, J. Wei, M. Jaime, F. Balakirev, D. A. Tennant, J. F. DiTusa, I. Chiorescu, L. Spinu, and Z. Q. Mao, "A magnetic topological semimetal $\mathrm{Sr}_{1-y} \mathrm{Mn}_{1-z} \mathrm{Sb}_{2}(y, z<0.1)$," Nat. Mater. 16, 905-910 (2017)

38 S. V. Ramankutty, J. Henke, A. Schiphorst, R. Nutakki, S. Bron, G. Araizi-Kanoutas, S. K. Mishra, Lei Li, Y. K. Huang, T. K. Kim, M. Hoesch, C. Schlueter, T. L. Lee, A. de Visser, Zhicheng Zhong, Jasper van Wezel, E. van Heumen, and M. S. Golden, "Electronic structure of the candidate 2D Dirac semimetal $\mathrm{SrMnSb}_{2}$ : A combined experimental and theoretical study," SciPost Phys. 4, 010 (2018)

sy Yong Liu, Tao Ma, Lin Zhou, Warren E. Straszheim, Farhan Islam, Brandt A. Jensen, Wei Tian, Thomas Heitmann, R. A. Rosenberg, J. M. Wilde, Bing Li, Andreas Kreyssig, Alan I. Goldman, B. G. Ueland, Robert J. McQueeney, and David Vaknin, "Crystal growth, microstructure, and physical properties of $\mathrm{SrMnSb}_{2}$," Phys. Rev. B 99, 054435 (2019).

40 Qiang Zhang, Satoshi Okamoto, Matthew B. Stone, Jinyu Liu, Yanglin Zhu, John DiTusa, Zhiqiang Mao, and David Alan Tennant, "Influence of magnetism on Dirac semimetallic behavior in nonstoichiometric $\mathrm{Sr}_{1-y} \mathrm{Mn}_{1-z} \mathrm{Sb}_{2}(y \sim 0.07 ; z \sim 0.02)$." Phys. Rev. B 100, 205105 (2019)

${ }^{41}$ Jinyu Liu, Jin $\mathrm{Hu}$, Huibo Cao, Yanglin Zhu, Alyssa Chuang, D. Graf, D. J. Adams, S. M. A. Radmanesh, L. Spinu, I. Chiorescu, and Zhiqiang Mao, "Nearly massless Dirac fermions hosted by Sb square net in BaMnSb 2 ," Sci. Rep. 6, 30525 (2016)

42 Silu Huang, Jisun Kim, W. A. Shelton, E. W. Plummer, and Rongying Jin, "Nontrivial Berry phase in magnetic $\mathrm{BaMnSb}_{2}$ semimetal," Proc. Natl Acad. Sci. USA 114, 6256-6261 (2017). 
43 Robert Kealhofer, Sooyoung Jang, Sinéad M. Griffin, Caolan John, Katherine A. Benavides, Spencer Doyle, T. Helm, Philip J. W. Moll, Jeffrey B. Neaton, Julia Y. Chan, J. D. Denlinger, and James G. Analytis, "Observation of a two-dimensional Fermi surface and Dirac dispersion in YbMnSb 2 ," Phys. Rev. B 97, 045109 (2018)

44 Yi-Yan Wang, Sheng Xu, Lin-Lin Sun, and Tian-Long Xia, "Quantum oscillations and coherent interlayer transport in a new topological Dirac semimetal candidate $\mathrm{YbMnSb}_{2}$," Phys. Rev. Mater. 2, 021201 (2018).

45 Xiangang Wan, Ari M. Turner, Ashvin Vishwanath, and Sergey Y. Savrasov, "Topological semimetal and Fermiarc surface states in the electronic structure of pyrochlore iridates," Phys. Rev. B 83, 205101 (2011).

46 J.-Z. Ma, S. M. Nie, C. J. Yi, J. Jandke, T. Shang, M. Y. Yao, M. Naamneh, L. Q. Yan, Y. Sun, A. Chikina, V. N. Strocov, M. Medarde, M. Song, Y.-M. Xiong, G. Xu, W. Wulfhekel, J. Mesot, M. Reticcioli, C. Franchini, C. Mudry, M. Müller, Y. G. Shi, T. Qian, H. Ding, and M. Shi, "Spin fluctuation induced Weyl semimetal state in the paramagnetic phase of $\mathrm{EuCd}_{2} \mathrm{As}_{2}$," Science Advances 5, eaaw4718 (2019)

47 Hongming Weng, Chen Fang, Zhong Fang, B. Andrei Bernevig, and Xi Dai, "Weyl semimetal phase in noncentrosymmetric transition-metal monophosphides," Phys. Rev. X 5, 011029 (2015).

48 Su-Yang Xu, Ilya Belopolski, Nasser Alidoust, Madhab Neupane, Guang Bian, Chenglong Zhang, Raman Sankar, Guoqing Chang, Zhujun Yuan, Chi-Cheng Lee, Shin-Ming Huang, Hao Zheng, Jie Ma, Daniel S. Sanchez, BaoKai Wang, Arun Bansil, Fangcheng Chou, Pavel P. Shibayev, Hsin Lin, Shuang Jia, and M. Zahid Hasan, "Discovery of a Weyl fermion semimetal and topological Fermi arcs," Science 349, 613-617 (2015)

49 B. Q. Lv, N. Xu, H. M. Weng, J. Z. Ma, P. Richard, X. C. Huang, L. X. Zhao, G. F. Chen, C. E. Matt, F. Bisti, V. N. Strocov, J. Mesot, Z. Fang, X. Dai, T. Qian, M. Shi, and H. Ding, "Observation of Weyl nodes in TaAs," Nat. Phys. 11, 724-727 (2015)

so Anmin Zhang, Changle Liu, Changjiang Yi, Guihua Zhao, Tian-long Xia, Jianting Ji, Youguo Shi, Rong Yu, Xiaoqun Wang, Changfeng Chen, and Qingming Zhang, "Interplay of Dirac electrons and magnetism in $\mathrm{CaMnBi}_{2}$ and $\mathrm{SrMnBi}_{2}, "$ Nat. Commun. 7, 13833 (2016)

51 A. Sapkota, L. Classen, M. B. Stone, A. T. Savici, V. O. Garlea, Aifeng Wang, J. M. Tranquada, C. Petrovic, and I. A. Zaliznyak, "Signatures of coupling between spin waves and Dirac fermions in YbMnSb 2 ," Phys. Rev. B 101, 041111(R) (2020)

${ }^{2}$ Ryoichi Kajimoto, Mitsutaka Nakamura, Yasuhiro Inamura, Fumio Mizuno, Kenji Nakajima, Seiko OhiraKawamura, Tetsuya Yokoo, Takeshi Nakatani, Ryuji Maruyama, Kazuhiko Soyama, Kaoru Shibata, Kentaro Suzuya, Setsuo Sato, Kazuya Aizawa, Masatoshi Arai, Shuichi Wakimoto, Motoyuki Ishikado, Shinichi Shamoto, Masaki Fujita, Haruhiro Hiraka, Kenji Ohoyama, Kazuyoshi Yamada, and Chul-Ho Lee, "The Fermi Chopper Spectrometer 4SEASONS at J-PARC," J. Phys. Soc. Jpn. 80, SB025 (2011)

53 Mark V. Kartsovnik, "High magnetic fields: A tool for studying electronic properties of layered organic metals," Chem. Rev. 104, 5737-5782 (2004)

54 G. P. Mikitik and Yu. V. Sharlai, "Manifestation of Berry's phase in metal physics," Phys. Rev. Lett. 82, 2147-2150
(1999)

55 Zhen Wang, Yi Zheng, Zhixuan Shen, Yunhao Lu, Hanyan Fang, Feng Sheng, Yi Zhou, Xiaojun Yang, Yupeng Li, Chunmu Feng, and Zhu-An Xu, "Helicity-protected ultrahigh mobility Weyl fermions in NbP," Phys. Rev. B 93, 121112 (2016)

56 Ratnadwip Singha, Arnab Kumar Pariari, Biswarup Satpati, and Prabhat Mandal, "Large nonsaturating magnetoresistance and signature of nondegenerate Dirac nodes in ZrSiS," Proc. Natl Acad. Sci. USA 114, 2468-2473 (2017).

57 A. Narayanan, M. D. Watson, S. F. Blake, N. Bruyant, L. Drigo, Y. L. Chen, D. Prabhakaran, B. Yan, C. Felser, T. Kong, P. C. Canfield, and A. I. Coldea, "Linear magnetoresistance caused by mobility fluctuations in $n$-Doped $\mathrm{Cd}_{3} \mathrm{~A}_{2}, "$ Phys. Rev. Lett. 114, 117201 (2015).

58 Yanfei Zhao, Haiwen Liu, Chenglong Zhang, Huichao Wang, Junfeng Wang, Ziquan Lin, Ying Xing, Hong Lu, Jun Liu, Yong Wang, Scott M. Brombosz, Zhili Xiao, Shuang Jia, X. C. Xie, and Jian Wang, "Anisotropic Fermi surface and quantum limit transport in high mobility three-dimensional Dirac semimetal $\mathrm{Cd}_{3} \mathrm{As}_{2}$," Phys. Rev. X 5, 031037 (2015).

${ }^{59}$ H. Murakawa, M. S. Bahramy, M. Tokunaga, Y. Kohama, C. Bell, Y. Kaneko, N. Nagaosa, H. Y. Hwang, and Y. Tokura, "Detection of Berry's phase in a bulk Rashba semiconductor," Science 342, 1490-1493 (2013).

60 Yoichi Ando, "Topological insulator materials," J. Phys. Soc. Jpn. 82, 102001 (2013)

${ }^{61}$ S. O. Diallo, V. P. Antropov, T. G. Perring, C. Broholm, J. J. Pulikkotil, N. Ni, S. L. Bud'ko, P. C. Canfield, A. Kreyssig, A. I. Goldman, and R. J. McQueeney, "Itinerant magnetic excitations in antiferromagnetic $\mathrm{CaFe}_{2} \mathrm{As}_{2}$," Phys. Rev. Lett. 102, 187206 (2009)

62 R. A. Ewings, T. G. Perring, J. Gillett, S. D. Das, S. E. Sebastian, A. E. Taylor, T. Guidi, and A. T. Boothroyd, "Itinerant spin excitations in $\mathrm{SrFe}_{2} \mathrm{As}_{2}$ measured by inelastic neutron scattering," Phys. Rev. B 83, 214519 (2011).

63 S Toth and B Lake, "Linear spin wave theory for single- $Q$ incommensurate magnetic structures," J. Phys.: Conden. Matter 27, 166002 (2015).

${ }^{04}$ G. L. Squires, Introduction to the Theory of Thermal Neutron Scattering (Dover, Mineola, NY, 1996).

65 G. Shirane, S. M. Shapiro, and J. M. Tranquada, Neutron Scattering with a Triple-Axis Spectrometer: Basic Techniques (Cambridge University Press, Cambridge, 2002).

66 Noam Morali, Rajib Batabyal, Pranab Kumar Nag, Enke Liu, Qiunan Xu, Yan Sun, Binghai Yan, Claudia Felser, Nurit Avraham, and Haim Beidenkopf, "Fermi-arc diversity on surface terminations of the magnetic Weyl semimetal $\mathrm{Co}_{3} \mathrm{Sn}_{2} \mathrm{~S}_{2}$," Science 365, 1286-1291 (2019).

67 D. F. Liu, A. J. Liang, E. K. Liu, Q. N. Xu, Y. W. Li, C. Chen, D. Pei, W. J. Shi, S. K. Mo, P. Dudin, T. Kim, C. Cacho, G. Li, Y. Sun, L. X. Yang, Z. K. Liu, S. S. P. Parkin, C. Felser, and Y. L. Chen, "Magnetic Weyl semimetal phase in a kagomé crystal," Science 365, 1282-1285 (2019)

${ }^{\circ}$ Qiunan Xu, Enke Liu, Wujun Shi, Lukas Muechler, Jacob Gayles, Claudia Felser, and Yan Sun, "Topological surface Fermi arcs in the magnetic Weyl semimetal $\mathrm{Co}_{3} \mathrm{Sn}_{2} \mathrm{~S}_{2}$," Phys. Rev. B 97, 235416 (2018).

69 Enke Liu, Yan Sun, Nitesh Kumar, Lukas Muechler, Aili Sun, Lin Jiao, Shuo-Ying Yang, Defa Liu, Aiji Liang, Qiunan Xu, Johannes Kroder, Vicky Süß, Horst Borrmann, Chandra Shekhar, Zhaosheng Wang, Chuanying Xi, Wen- 
hong Wang, Walter Schnelle, Steffen Wirth, Yulin Chen, Sebastian T. B. Goennenwein, and Claudia Felser, "Giant anomalous Hall effect in a ferromagnetic kagomé-lattice semimetal," Nat. Phys. 14, 1125-1131 (2018).

${ }^{70}$ Qi Wang, Yuanfeng Xu, Rui Lou, Zhonghao Liu, Man Li, Yaobo Huang, Dawei Shen, Hongming Weng, Shancai Wang, and Hechang Lei, "Large intrinsic anomalous Hall effect in half-metallic ferromagnet $\mathrm{Co}_{3} \mathrm{Sn}_{2} \mathrm{~S}_{2}$ with magnetic Weyl fermions," Nat. Commun. 9, 3681 (2018)

${ }^{71}$ Ilya Belopolski, Kaustuv Manna, Daniel S. Sanchez, Guoqing Chang, Benedikt Ernst, Jiaxin Yin, Songtian S. Zhang, Tyler Cochran, Nana Shumiya, Hao Zheng, Ba- hadur Singh, Guang Bian, Daniel Multer, Maksim Litskevich, Xiaoting Zhou, Shin-Ming Huang, Baokai Wang, Tay-Rong Chang, Su-Yang Xu, Arun Bansil, Claudia Felser, Hsin Lin, and M. Zahid Hasan, "Discovery of topological Weyl fermion lines and drumhead surface states in a room temperature magnet," Science 365, 1278-1281 (2019)

T2 Pyeongjae Park, Joosung Oh, Klra Uhlírová, Jerome Jackson, Andrs Dek, L szl Szunyogh, Ki Hoon Lee, Hwanbeom Cho, Ha-Leem Kim, Helen C. Walker, Devashibhai Adroja, Vladimr Sechovsky, and Je-Geun Park, "Magnetic excitations in non-collinear antiferromagnetic Weyl semimetal $\mathrm{Mn}_{3} \mathrm{Sn}, "$ npj Quant. Mater. 3, 63 (2018). 